\title{
LMENTAL. UN PROCEDIMIENTO MENTAL SIMPLE PARA LA DERIVACIÓN DE SISTEMAS COMPLEJOS DE RELACIONES SINTÁCTICAS
}

\author{
GuILLERMO LORENZO GONZÁleZ \\ Universidad de Oviedo. Área de Lingüística General \\ glorenzo@uniovi.es \\ Luis Polo Paredes \\ Fundación CTIC. I+D+I \\ Luis.polo@fundacionctic.org
}

\begin{abstract}
Resumen
Este trabajo desarrolla un procedimiento enormemente simple para la generación de sistemas complejos de relaciones. Se propone como un modelo del procedimiento mental empleado en la formación y transformación de estructuras de frase en las gramáticas naturales. Sostenemos que una parte importante de los problemas planteados por la teoría desnuda de la estuctura de frase del Programa Minimalista pueden ser superados gracias a este procedimiento. Proponemos además un primer conjunto de aplicaciones mediante el análisis de ciertos tipos frásticos y de los fenómenos ligados a ellos.

PALABRAS CLAVE: estructura de frase, movimiento, derivación, niveles de representación, Programa Minimalista.
\end{abstract}

\begin{abstract}
This paper suggests a very simple procedure capable of generating complex systems of relationships. It is proposed as a model of the mental procedure used in the formation and transformation of phrase structures within natural grammars. We claim that several problems of the minimalist bare phrase structure theory can be surmounted by using our model. We also propose a first set of applications to the analysis of certain phrasal types and related phenomena.
\end{abstract}

KEY WORDS: Phrase structure, movement, derivation, levels of representation, Minimalist Program.

\section{Introducción}

Las pretensiones de nuestro trabajo son ambiciosas, pero el alcance de esta exposición inicial de nuestras ideas tendrá que ser bastante limitado. Buscamos el desarrollo de un procedimiento combinatorio en extremo simple, pero capaz de derivar sistemas altamente complejos de relaciones sintácticas. Aspiramos, además, a que sea un modelo adecuado del operativo mental encargado de la computación de frases y oraciones en las lenguas naturales. Comenzaremos exponiendo las propiedades formales básicas de tal procedimiento combinatorio o computacional, al que denominaremos «LMental» precisamente para dar idea, en primer lugar, de su extrema simplicidad y, en segundo lugar, de nuestra pretensión de elevarlo al rango de modelo del sistema de computación sintáctica del que hace uso la mente humana. A continuación mostraremos su capacidad para derivar el tipo de propieda- 
des tradicionalmente asociadas a las operaciones de movimiento sin necesidad de aplicar desplazamiento alguno de constituyentes ni de recurrir a otros mecanismos que repliquen sus efectos. Finalmente, lo aplicaremos al análisis de un fenómeno, el de la regulación de las posibilidades de alcance mutuo entre cuantificadores, que habitualmente implica la postulación de niveles sucesivos de representación, aunque de nuevo sin la necesidad de operar sobre niveles de representación añadidos.

Somos conscientes del carácter altamente tentativo de algunas de nuestras especulaciones y del alcance aún muy limitado de nuestras aplicaciones empíricas. Con todo, creemos que se trata de un importante primer paso de cara a la elaboración de un modelo gramatical capaz de llevar a término un buen número de los ideales teóricos del minimalismo chomskyano, si bien sobre la base de herramientas de análisis muy diferentes a las consideradas "estándar" en el contexto del Programa Minimalista. En concreto, creemos que nuestro modelo es extremamente fiel al patrón teórico minimalista en, al menos, tres aspectos fundamentales:

- la apelación a recursos de la mayor simplicidad y generalidad posibles,

- la eliminación de aquellos con una motivación estrictamente gramatical (entre los que Chomsky suele poner el "desplazamiento" de constituyentes como ejemplo), y

- la preferencia por una arquitectura monoestratal que limite los interfaces de la facultad del lenguaje a aquellos que necesariamente mantiene con los sistemas externos o de actuación a los que sirve de vía de contacto (véase Chomsky 1995a y 2000 para una explicitación de los principios fundamentales del minimalismo).

Por todas estas razones, estimamos que vale la pena explorar hasta las últimas consecuencias el sistema de generación de relaciones sintácticas que avanzamos en este trabajo.

\section{Mecanismo y propiedades básicas de LMental: 1. "Ensamblaje" y "dominio estruc- tural"}

LMental es, en esencia, un procedimiento sintáctico de extrema simplicidad, cuyo mecanismo se limita a una operación de "ensamblaje" (E) que actúa del siguiente modo:

(1) $E(a, b)=(a(b))$

Se basa, por tanto, en la aplicación de "operaciones binarias" cuyo efecto es el establecimiento de una "relación asimétrica" entre los elementos ensamblados. E1 carácter binario de las operaciones y la naturaleza asimétrica de los resultados remiten, evidentemente, al modelo de «estructura desnuda de frase» de Chomsky (1995b y trabajos subsiguientes). No obstante, queremos resaltar algunas diferencias cruciales entre dicho modelo y el que aquí proponemos.

Debe quedar claro, ante todo, que nuestro modelo no "proyecta" asimétricamente como el chomskyano, en el sentido de que uno u otro de los elementos relacionados (pero en ningún caso los dos o partes de uno y otro) "etiquete" el nuevo nivel de estructura resultante de la aplicación de E. En nuestro caso, lo que resulta de E no es un nuevo nivel de estructura, sino una nueva relación, "asimétrica" porque los elementos que la contraen no participan en ella en idénticas condiciones. Nosotros llamamos a esa relación "dominio estructural" (Dom) y en ella, por definición, un elemento cuenta como "dominador" (D) y el otro como 
"dominado" (d). No hay situaciones de igualdad o de ausencia de dominio, de modo que nos atenemos al "principio de exocentricidad" generalmente asumido en los diferentes modelos generativos.

Resulta posible representar tanto la operación (E) como la relación resultante (Dom) a través de un esquema arbóreo como (2):

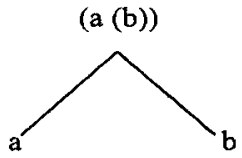

En adelante, sin embargo, evitaremos este sistema de representación porque resulta equívoco a la hora de reflejar el funcionamiento y propiedades que nosotros atribuimos a la sintaxis. Insistimos en que, desde nuestro punto de vista, resulta crucial la idea de que el resultado de cada nueva aplicación de $\mathrm{E}$ no da lugar (ni sirve de etiqueta) a un nuevo nivel de estructura, sino que constituye la única estructura relevante a efectos sintácticos. En otras palabras, la nueva relación (o sistema de relaciones) a que ha dado lugar $E$ agota todos los detalles sintácticamente relevantes o activos en un determinado momento de la derivación de una estructura lingüística. Podemos también expresarlo diciendo que las ramas de los esquemas arbóreos y cuanto queda por debajo de ellas carecen en nuestro modelo de estatuto alguno. Por tanto, ciñéndonos al punto de partida de una derivación, como el que se corresponde con (2), todo lo anterior significa que "(a (b))" no guarda ninguna relación de dominio con "a" y con "b", ya que la única relación existente es la que "a" (D) mantiene con "b" (d). Por razones que abajo resultarán más claras, decimos que sólo "a" se relaciona con "b" (pues lo "domina") o, lo que es lo mismo, que "ser dominado" significa no entrar en relación con ningún otro elemento. Por eso, pensamos que el sistema de representación más acorde con todo lo dicho es un grafo como el que aparece en (3):

(3)

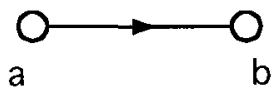

Una relación como la establecida a través de (1) y representada en (3) cuenta a su vez como uno de los elementos sobre los que resulta posible una nueva aplicación de $\mathrm{E}$, tal y como se formula en (4):

(4) $\mathrm{E}(\mathrm{c},(\mathrm{a}(\mathrm{b})))=\{(\mathrm{a}(\mathrm{b}))\{\mathrm{c}\}\}^{1}$

En esta nueva aplicación de E, "(a (b))" cuenta como "dominador" (D) y "c" como "dominado" (d). Por tanto, y según lo establecido, "c" no contrae relación alguna en el sistema de relaciones recién establecido; en cambio, "b", que no había contraído ninguna hasta el momento, pasa a contraerla con "c" en virtud de la relación de "dominio estructural" (Dom) 
que "(a (b))" contrae con "c". Recurriendo de nuevo al sistema de representación mediante grafos, el sistema de relaciones resultante es el siguiente:

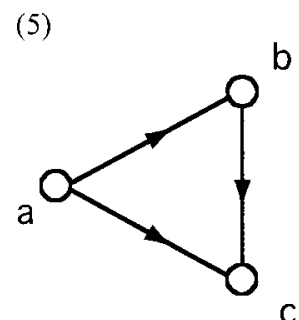

Lo que (5) nos permite apreciar es que el mecanismo sintáctico que manejamos ha conseguido, en este punto de la derivación, establecer tres relaciones diferentes mediante dos aplicaciones de $\mathrm{E}$, dos de ellas nuevas y una ya establecida mediante en la primera aplicación de E:

a) relaciones viejas (o ya establecidas mediante $E_{1}$ ): (a (b));

b) relaciones nuevas (o recién establecidas mediante $E_{2}$ ): (a (c)) y (b (c)).

Debe tenerse en cuenta que un grafo es una estructura recursiva, de manera que (5), resultado de la aplicación de $\mathrm{E}_{2}$, debe entenderse como una estructura que contiene como una de sus partes la estructura (3), resultado de la aplicación de $E_{1}$.

Creemos que en este punto quedan mejor marcadas y podemos dejar mejor establecidas las diferencias entre los sistemas de relaciones que se establecen mediante nuestro mecanismo de ensamblaje sintáctico con relación a los que establecería el mecanismo chomskyano. Para apreciarlo, conviene volver a la convención arbórea de representación. En el mismo punto de la derivación al que nosotros hemos llegado, el mecanismo chomskyano habría alcanzado el siguiente nivel de estructura:

(6)

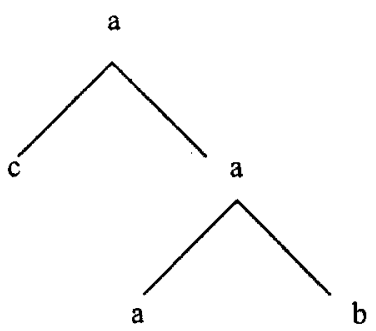

En esta representación, "a" mantiene una relación de dominio sobre el resto de la estructura, adquirida en función de la capacidad de proyección sucesiva que le confiere su condición de núcleo. Respecto a este aspecto nuestro sistema, en el que "a" es el único elemento que actúa como dominador con relación al resto, no sería más que una variante notacional del chomskyano. Sin embargo, en el modelo chomskyano los elementos adquieren propiedades en función de la posición que ocupan en la geometría arbórea, las cuales carecen en cambio, desde nuestro punto de vista, de cualquier relevancia (son, de hecho, inexistentes a todos los efectos). 
Una diferencia clave que se sigue de lo anterior es que, en el modelo chomskyano, "c" tiene un rango superior al de "b" debido a que "b", pese a no estar dominado por "c", lo está por todos los nodos que dominan a éste, mientras que "c" queda fuera del dominio de uno de los nodos que dominan a "b" (o traducido a la jerga derivacional de, por ejemplo, Epstein et al. 1998, "c" tiene un rango estructural superior al de "b" porque ha entrado más tarde en la derivación, de modo que el rango resulta ser un reflejo o una función del "timing" del proceso de derivación de las estructuras). Para nosotros, en cambio, "c" no ha contraído hasta este punto ninguna relación con los demás elementos sobre los que ha operado $\mathrm{E}$. No concedemos estatuto sintáctico alguno, tal y como establecimos más arriba, ni a las ramas ni a cuanto queda por debajo de éstas y, por tanto, los elementos que participan en un sistema de relaciones no pueden adquirir propiedades de la geometría arbórea (ni del "timing" del proceso mediante el que se ha derivado). En una representación como (7), nuestra alternativa arbórea a (6), todas las propiedades sintácticas efectivas están dadas en el nivel superior de la representación y, en éste, "c" cuenta como el único elemento no relacionado con ningún otro. Los niveles y elementos inferiores deben ser únicamente vistos como una especie de registro de las computaciones que han dado lugar al sistema de relaciones reflejado en el nivel superior y es totalmente irrelevante a efectos sintácticos (razón por la que nos inclinamos por evitar este tipo de representación en favor de los grafos):

(7) $\{(\mathrm{a}(\mathrm{b}))\{\mathrm{c}\}\}$

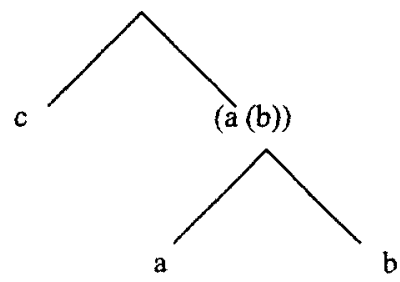

Es evidente, por tanto, que nuestro procedimiento sintáctico no es una simple variante notacional del chomskyano. En éste, "c" goza de una superioridad estructural sobre "b" de la que carece de acuerdo con nuestro sistema; $y$, a la inversa, "b" cuenta para nosotros como dotado de superioridad estructural respecto a "c". Más adelante evaluaremos empíricamente este tipo de diferencias.

Entre tanto, nos gustaría señalar algunas virtudes de orden teórico que por el momento cabe ya conceder a LMental. En un trabajo reciente muy influyente, Collins (2002) ha señalado que la asunción de la tesis minimalista más fuerte obliga, entre otras cosas, a la elaboración de una teoría de la estructura de frase que evite el "etiquetado" de los resultados de la operación de ensamblaje. Desde su punto de vista, cuanto cabe exigir al sistema computacional de una gramática es que provea los medios, necesariamente finitos, aptos para dar lugar a una capacidad combinatoria sin embargo infinita. Pero para ello, razona Collins (2002: 43) no se ve el porqué de que la aplicación de cada nueva combinación deba verse acompañada de la necesidad de escoger uno u otro de los elementos combinados como etiqueta de la frase resultante. Desde este punto de vista, Collins (2002: 42) señala que un procedimiento combinatorio que propicie resultados como los de (9) debería ser preferido, bajo los supuestos del minimalismo, frente a otro que de lugar a resultados como los de (8): 
(8)

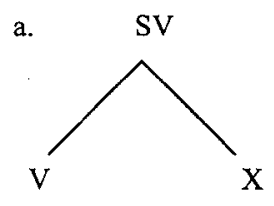

(9) a.

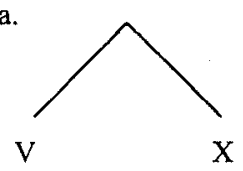

b. $(\mathrm{V}(\mathrm{V}, \mathrm{X}))$

b. $(\mathrm{V}, \mathrm{X})$

Pues bien, el tipo de relación básica que LMental genera se aproxima mucho más al tipo de (9) que al de (8). Es cierto que esta observación puede verse oscurecida por el hecho de que la relación representada en (9) no manifiesta, al contrario de las generadas por LMental, una dirección definida de dominio entre sus miembros, la cual pudiera además verse como innecesaria desde el punto de vista de los requerimientos (mínimos) a los que una gramática ha de ajustarse ${ }^{2}$. Sin embargo, y sobre ello volveremos más adelante, es razonable suponer que dicha dirección de dominio es siempre reflejo de imperativos de orden conceptual o semántico y, como tal, una muestra más de ajuste gramatical a las exigencias de los sistemas externos.

Por otro lado, Collins (2002: 44 y 49) observa que el sistema de etiquetado propio tanto de la teoría $X^{\prime}$ como de la estructura desnuda de frase representa una auténtica rémora con relación a la aspiración de alcanzar un modelo gramatical lo más radicalmente derivacional que sea posible. En palabras del propio Collins (aunque traducidas por nosotros): "Una etiqueta, una vez creada, se mantiene en existencia durante el resto de la derivación como parte de la representación que está siendo continuamente construida. Una etiqueta es accesible a lo largo de la derivación y en los interfaces" (Collins 2002: 49). Esta "persistencia" (Collins 2002: 44) de las etiquetas, que se mantienen activas mucho más allá de lo que en todo caso podría estar justificado, no sólo atenúa la naturaleza derivacional del modelo gramatical, sino que choca además frontalmente con los supuestos de economía que el minimalismo defiende.

2 Chomsky parece tomar partido por esta interpretación al afirmar que "la operación básica [del sistema computacional] será la más simple posible: ensamblaje no estructurado, formando un conjunto" (Chomsky 2005: 15). Esto significa que dados dos elementos léxicos cualesquiera, " $a$ " y "b", la aplicación de la operación dará lugar al conjunto $\{a, b\}$, sin asimetria estructural alguna manifiesta en el objeto sintáctico. Toda posible asimetría se derivará de las propiedades semánticas de los elementos que participan en él. Esta posición no nos parece convincente por una razón fundamental. El hecho de disponer de un conjunto, como $\{\mathrm{a}, \mathrm{b}\}$, no garantiza que se registren relaciones entre sus miembros. Por tanto, desde la óptica de Chomsky:

- o bien las relaciones se introducen a posteriori;

- o bien la asimetría del conjunto radica en que contiene todas las relaciones posibles entre los elementos (en nuestro caso, $\{a \rightarrow b\}$ y $\{a \leftarrow b\}$ ), de modo que la semántica pueda escoger en cada caso la relación cuyo sentido más le convenga.

El problema es que la primera aiternativa viola el "principio de inclusividad" (Chomsky 1995a: 228) y con la segunda el sistema está manejando de entrada relaciones conceptualmente innecesarias (en nuestro caso, \{a $\leftarrow$ b) ). Por todo esto, somos partidarios de mantener la idea del objeto sintáctico como "par ordenado", con el orden (el sentido de la relación) determinado externamente por las propiedades semánticas de los elementos, pero con el sistema computacional ajustado para responder a este tipo de exigencias externas. 
Ninguno de estos problemas se plantea con LMental. Debe apreciarse, en primer lugar, que los sistemas de relaciones sintácticas se obtienen con LMental mediante la aplicación sucesiva de la operación E y, en segundo lugar, que lo que se va obteniendo es una única estructura en la que las relaciones proliferan a medida que se suman nuevas aplicaciones de E. Por tanto, el procedimiento combinatorio no va dejando tras de sí una estela residual de relaciones etiquetadas. No olvidemos que el tipo de representación gráfica que verdaderamente capta el funcionamiento de este sistema son grafos como los que aparecen en (3) y en (5), que repetimos (y completamos al modo de Collins) como (10) y (11) respectivamente:

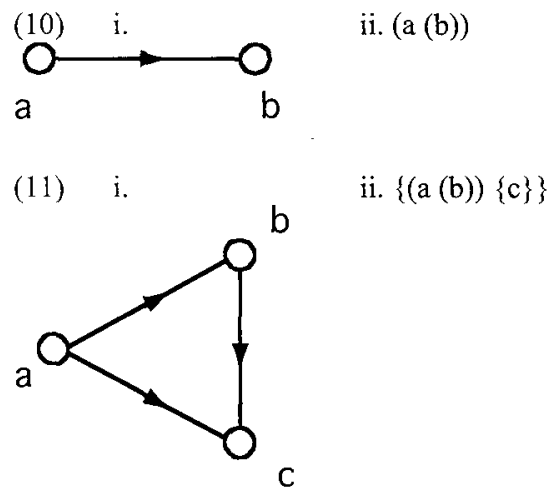

Tenemos buenas razones para pensar, en definitiva, que LMental es un candidato ciertamente prometedor para profundizar, desde la óptica minimalista, en una modelización realista del procedimiento del que la mente humana hace uso para generar sistemas complejos de relaciones sintácticas.

\section{Mecanismo y propiedades básicas de LMental: 2. "Cadenas" y "mallas"}

Nos interesa comenzar a visualizar la operatividad de LMental sobre materiales lingüísticos concretos. Nos situaremos, para ello, en un estadio derivacional correspondiente al representado en (11). Hasta ese punto, tal y como explicamos arriba, el sistema ha dado lugar al establecimiento de tres relaciones, cada una de las cuales sirve como cauce formal para la plasmación de una determinada relación de tipo gramatical:

1. (a (b)), por ejemplo, puede servir para la asignación de rol argumental por parte de un núcleo predicativo (D) sobre su complemento (d):

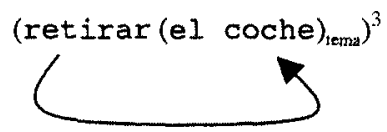

3 Con el cambio en el tipo de letra queremos dar a entender que los elementos sobre los que actúa $\mathrm{E}$ son piezas tomadas del léxico sea cual sea el formato representacional con que allí están presentes. Queremos evitar, en definitiva, cuestiones como la de si se trata de elementos flexionados o no flexionados o la del grado de precisión de las instrucciones fónicas asociadas a ellos. 
2. (a (c)), por su parte, puede servir para la asignación por parte de ese mismo núcleo (D) de un segundo rol, en este caso, sobre el especificador (d) ${ }^{4}$ :

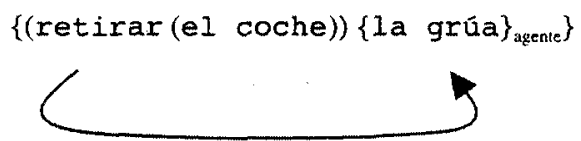

3. (b (c)), finalmente, puede servir para señalar al complemento (D) como posible antecedente del especificador (d) a efectos referenciales:

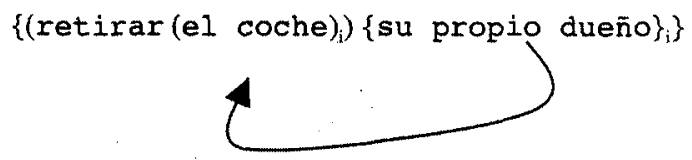

Teniendo estas tres relaciones a la vista, podemos pasar ahora a establecer y justificar una importante diferencia entre, de un lado, las relaciones 1 y 2 -es decir, (a (b)) y (a (c))- y, de otro lado, la relación 3 -o sea, (b (c)) - , algo crucial, como veremos, para nuestro modelo. Como se ha podido apreciar, las relaciones (a (b)) y (a (c)) han sido aprovechadas para establecer las relaciones semánticas de "tema" y "agente" entre el "dominador" (que asigna tales papeles) y los "dominados" (que los reciben). En ambos casos, la sintaxis ha sido utilizada para encauzar relaciones de tipo semántico previstas en el propio significado del dominador. En adelante, denominaremos "cadenas" a las relaciones sintácticas que sirven a tal fin (es decir, el de dar satisfacción a relaciones de orden conceptual impuestas por el dominador) y "eslabones" a los elementos que las contraen. Desde este punto de vista, (a (b)) y (a (c)) son por tanto dos cadenas que comparten uno de sus eslabones (en concreto, el eslabón dominador de ambas relaciones); en buena lógica, podemos entender que componen una única cadena como la que simboliza el grafo (15):

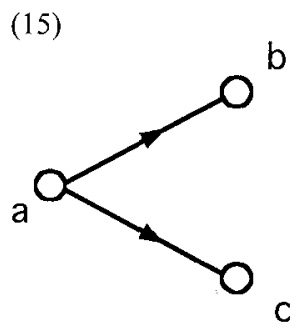

(15) no es, sin embargo, un objeto sintáctico posible en LMental. La razón es simple: desde el momento mismo en que se establece la relación (a (c)), LMental establece también automáticamente la relación (b (c)). Es decir; LMental no puede dar lugar a un grafo como (15) sin generar automáticamente una estructura como (16):

4 Utilizamos términos como "especificador", “adjunto" o similares como simples etiquetas deseriptivas. Carecen, desde la perspectiva de LMental, de cualquier valor teórico. 
(16)

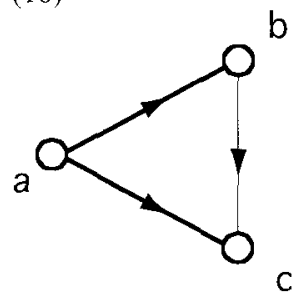

Es interesante apreciar que (a (b)) y (a (c)) obedecen a un tipo de motivación muy diferente a la de (b (c)) en los ejemplos (12) a (14): las primeras obedecen a imperativos de orden semántico dictados por el dominador, mientras que la última es una consecuencia o efecto de una capacidad de sobregeneración propia del procedimiento combinatorio. Pero lo interesante es que la gramática de las lenguas naturales parece hacer uso de este tipo de relaciones sobregeneradas para encauzar relaciones semánticas (como la de "antecedente") que carecen del carácter imperativo de, por ejemplo, los roles argumentales determinados por la semántica de los núcleos predicativos.

Para captar mejor todo lo anterior proponemos establecer una distinción esencial entre la noción de "cadena", ya introducida, y la noción de "malla". Una "malla" es el tipo de estructura relacional que resulta del establecimiento de al menos dos cadenas. Esto significa que al "encadenar" cadenas lo que se obtiene no es otra cadena más compleja, sino un nuevo tipo de estructura: una "malla", la cual consiste en las relaciones introducidas por una serie de encadenamientos - en el caso de (16), (a (b)) y (a (c)) - más las relaciones sobregeneradas automáticamente por el proceder característico del sistema - (b (c)), en el caso de (16)--.

Procedamos pues a ensamblar un nuevo elemento "d" a la estructura (16); el resultado será una estructura como lo que representa el grafo (18):

$$
E(d,\{(a(b))\{c\}\})=[d[\{(a(b))\{c\}\}]]
$$

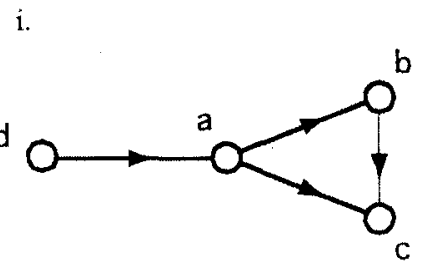

ii. $[d[\{(\mathrm{a}(\mathrm{b}))\{\mathrm{c}\}\}]]$

De acuerdo con la mecánica del modelo, de esta nueva aplicación de E ha resultado la introducción de tres nuevas relaciones que, sumadas a las tres anteriormente existentes, da lugar a un total del seis relaciones:

a) relaciones viejas (heredadas de $E_{2}$ ): (a (b)), (a (c)) y (b (c));

b) relaciones nuevas (introducidas mediante $\left.E_{3}\right)$ : (d (a)), (d (b)) y (d (c)).

En este punto, además, "d" cuenta como el elemento más relacionado del sistema (es triplemente D), mientras que "c" cuenta como el menos relacionado de todos (de hecho, 
no contrae ninguna relación); "a" contrae dos relaciones y "b" una relación. En cuanto a la operatividad de las tres relaciones nuevas -(d (a)), (d (b)) y (d (c)) - , pueden servir, por ejemplo, para encauzar las relaciones de "alcance" ejercitadas por un elemento (por ejemplo, un adjunto de nivel verbal) sobre todos los demás insertados hasta ese punto: \\ [tal vezopi $[\{$ retirar (el coche)) \{su propio dueño\}\}]i]}

Volviendo a las nociones introducidas arriba, diremos que el "encadenamiento" de "d" con " $a$ " — (d (a) ) — ha dado lugar automáticamente a la siguiente trama de relaciones:

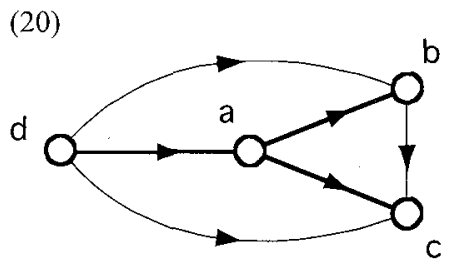

En la secuencia analizada arriba, "tal vez" ("d") es un "operador modal" que se encadena a un "predicado" ("retirar"; "a") por imperativo semántico —(d (a))—, de modo que adquiere automáticamente "alcance" sobre los elementos previamente encadenados a "a" - (d (b)) y (d (c)) - . El esquema que aparece en (20) explicita todas las relaciones que se obtienen espontáneamente al establecer las cadenas que los elementos "a" y " $d$ " se encuentran determinados a establecer. No obstante, todas ellas deben considerarse ya "dadas" en una representación como (18i), respecto a la cual (20) no es más que una variante notacional.

En las mallas se explicita, además de las relaciones sintácticas que obedecen a imperativos semánticos - como las cadenas (a (b)), (a (c)) y (d (a))—, una única relación sintáctica sobregenerada, es decir, no creada mediante encadenamiento semánticamente motivado - $(\mathrm{b}(\mathrm{c}))$ - . Esta relación, aunque sobregenerada, debe explicitarse en la malla con el objeto de señalar cuál es el elemento que no contrae ninguna relación (es decir, no domina a ningún otro elemento) y sirve así de punto de cierre al sistema de relaciones que se ha obtenido mediante las sucesivas aplicaciones de E. Esto significa que la malla no es simplemente una cadena compleja (es decir, un encadenamiento de cadenas), ya que incluye una "relación de cierre" que no es en sí misma una cadena. La malla es, no obstante, el tipo de estructura que realmente refleja los encadenamientos semánticamente motivados que, por el automatismo propio del sistema, van dando lugar a redes complejas de relaciones sintácticas que van siendo "colonizadas" por nuevos tipos de vínculos semánticos no imperativos 5 .

5 La distinción entre las relaciones locales (o "cadenas") y las relaciones sobregeneradas (que se siguen de la "transitividad" propia del sistema) en representaciones como (18i) resulta de la perspectiva teórica asumida en este trabajo. Un grafo, como tal, no es más que un conjunto de relaciones indiferenciadas, al que podemos denominar "red de relaciones de dominio estructural" (o, sencillamente, "red"). La "malla" es en cambio el objeto teórico que refleja la distinción, esencial para nuestro enfoque, entre los dos tipos de relaciones apuntados arriba. La "red" carece por tanto, para nosotros, de cualquier estatuto teórico. Véase, con relación a todo esto, el Anexo I. 


\section{Propiedades avanzadas de LMental: Derivando (sin movimiento) los efectos de "muévase $\alpha$ "}

Pasaremos ahora a tratar de explicar los efectos tradicionalmente atribuidos a la actuación de un mecanismo transformacional (y, habitualmente, a la aplicación de la operación de desplazamiento de constituyentes "muévase $\alpha$ ") haciendo uso de los mecanismos básicos de LMental y sin necesidad, por tanto, de desplazar elemento alguno de su posición original. Esto nos permitirá ver que, en realidad, los elementos sometidos a $E$ no ocupan posición alguna, lo que en definitiva resalta la naturaleza estrictamente derivacional de nuestro procedimiento computacional. En efecto, si podemos prescindir de la necesidad de atribuir posiciones de "origen" y de "destino" para los elementos que van siendo introducidos por E, parece claro que entonces deja de tener sentido la postulación de representaciones mentales que consignen y memoricen unas y otras posiciones con relación a cada una de las piezas computadas. En LMental cada pieza se introduce una sola vez como partícipe en una relación y a partir de ese momento no se somete a más alteraciones, en el sentido de que no se convierte en "diana" de ninguna otra operación computacional. Se va modificando, eso sí, el conjunto de relaciones en que participa, como resultado natural de las sucesivas aplicaciones de E sobre otros elementos directamente tomados del léxico. ${ }^{6}$

Para aclarar todo lo anterior necesitamos introducir previamente una noción semántica que denominaremos "estructura básica de localización" (en adelante, BL), la cual actúa como un determinante conceptual especialmente esencial sobre la sintaxis. Entendemos por BL el dispositivo semántico a través del cual una relación predicativa (en principio un "tipo" semántico abstracto) pasa a ser expresión de una "instancia" o "ejemplar" particular de dicho tipo. Actúan como introductores de BLs los elementos auxiliares y morfológicos relacionados con el tiempo, el modo o el aspecto, valores que cumplen la función conceptual de aportar unas coordenadas referenciales para la instanciación del tipo. No obstante, la BL (en el sentido en que aquí la entendemos) implica asimismo la integración conceptual de uno de los argumentos (el "sujeto", en términos descriptivos) dentro de la coordenada referencial aportada. Por tanto, el introductor de una BL está sujeto a un doble imperativo semántico:

- de un lado, debe entrar en relación con un predicado;

- de otro lado, debe relacionarse en particular con el "argumento externo" de ese predicado.

Por tanto, de acuerdo con la mecánica de LMental, está llamado a encadenarse con dos elementos diferentes, lo que parece plantear un conflicto al procedimiento sintáctico, que

6 En cierto sentido podemos decir que LMental es, no exactamente un modelo estrictamente derivacional, sino un modelo de computación al que la distinción "derivacional" vs. "representacional" le resulta ajena. Como razonan Longa y Lorenzo (2001), todos los modelos gramaticales propuestos por el generativismo han participado de ambos aspectos y la caracterización en esos términos se basa en la prevalencia de uno u otro en cada caso. Así, si catalogamos el Modelo GB como "representacional" es porque las "condiciones de buena formación" afectan a las propiedades de los efectos de las computaciones en "locus" específicamente señalados al efecto (EP, ES, FL o FF); el Programa Minimalista se cataloga, en cambio, como "derivacional" porque dichas condiciones afectan a las computaciones mismas ( $o$, en todo caso, a las representaciones no lingüisticas resultantes). Puesto que LMental se basa exclusivamente en el efecto acumulativo de las relaciones entabladas entre los elementos tomados del léxico, parece que queda al margen de dichas distinciones. En todo caso, la "sucesividad" de las diferentes aplicaciones de E puede ser visto como un "residuo" derivacional del modelo que, en todo caso, no implica contrapartidas representacionales. 
opera exclusivamente mediante emparejamientos binarios. En principio, LMental podría generar una malla como la siguiente (en la que "e" sería el introductor de una BL, por ejemplo, el valor de "pasado"):

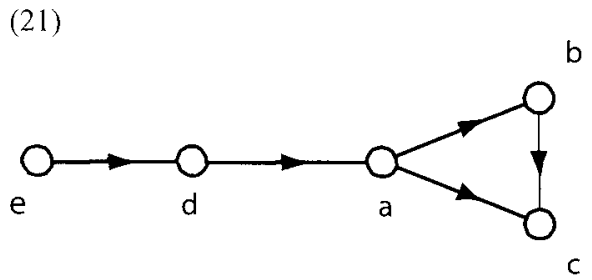

Con esta malla se da en efecto cumplimiento al primero de los imperativos semánticos al que está sujeto el introductor de la BL — "e"; "pasado"--, ya que conforma una cadena - (e (d (a)) ; (pasado (tal vez (retirar)))-cuyo eslabón dominado es un predicado — (d (a)); (tal vez (retirar) ) - . No olvidemos que si bien el predicado propiamente dicho es "retirar" - por tanto, "a"-, "tal vez" ("d") es un operador que no cambia el tipo semántico del predicado; por tanto, se cumple que el introductor de la BL _- "e"; "pasado" - se ha encadenado a un predicado - (d (a)); (tal vez (retirar)) - dando así satisfacción al primero de los imperativos semánticos a los que está sujeto. Por otra parte, en función de la capacidad de sobregeneración de relaciones propia del procedimiento, "d" pasa también a relacionarse con "b" - "el coche"-, tal cual se refleja de hecho en la malla representada en (2l), y con "c" — "su propio dueño"-, argumento externo del predicado, como se refleja en (22):

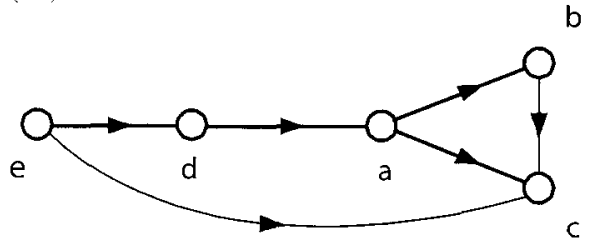

En principio, este sistema de relaciones parecería bastar para satisfacer los dos requisitos sintácticos a los que está sujeto "e" como introductor de una BL: por una parte, domina directamente a un predicado - (d (a) ) - y, por otra, domina indirectamente al argumento externo de ese predicado - "c" - en virtud de la transitividad de las relaciones de dominio - (d (a)) y (a (c)), por tanto, (d (c)) - . Sin embargo, LMental exige que las relaciones semánticas de tipo imperativo se establezcan localmente, por tanto, mediante una cadena simple, tal como se explicita en (23):

(23) Principio $\Gamma$ :

"Un imperativo semántico debe realizarse sintácticamente a través una cadena?."

7 El principio formulado por Chomsky (2000: 103) según el cual las relaciones temáticas debe establecerse mediante "ensamblaje puro" ("pure merge"), es decir, sin la asistencia de la operación de movimiento, queda por tanto subsumido bajo nuestro Principio $\Gamma$. 
Esto implica, pues, que la malla sintáctica representada en (21) no sirve para dar satisfacción a los imperativos semánticos a que está sujeto el introductor de una BL.

La alternativa es una malla en la que, como en (24), el introductor de la BL - "e"- - se ensamble a la estructura a través del elemento en relación con el cual puede dar satisfacción al segundo imperativo semántico —es decir, con " $\mathrm{c}$ "--, dando así lugar a la cadena (e (c)):

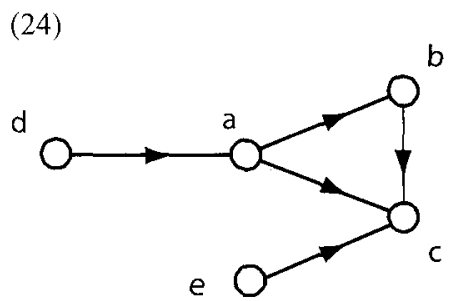

Es importante apreciar que a través de esta operación no se introduce en el sistema ninguna otra relación además de (e (c)), dado que " $\mathrm{c}$ " no contrae por su parte ninguna relación que pudiera dar lugar a otras por transitividad. Esto justifica que "e" pueda a continuación encadenarse con "d", como en (25), dando de este modo satisfacción al primer imperativo semántico al que está sujeto:

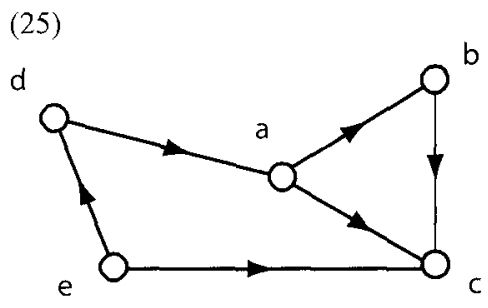

Es preciso valorar que el encadenamiento de "e" con "c" partiendo de una malla como (21) implicaría el establecimiento de una relación que, en realidad, ya estaría dada por transitividad en la propia malla - (e (d)), (d (a)) y (a (c)), por tanto (e (c)), como se aprecia en (22)--, lo que podemos entender como violación de los criterios de economía a que se sujeta LMental; en este caso concreto, del principio que podemos formular del siguiente modo:

(26) Principio $\Sigma$ :

"* $E(x, y)=(x(y))$, si $(x(y))$ ya está dada en la malla."

En cambio, el encadenamiento de "e" con "d" partiendo de una malla como (24) sirve para introducir una relación aún no existente en el sistema. Por tanto, los principios $\Gamma$ y $\Sigma$ “conspiran" para que (24) y (25) sean la única ruta derivacional posible para dar cumplimiento a los requisitos semánticos a los que debe responder el introductor de la $\mathrm{BL}$.

Volvamos al grafo (25). Del ensamblaje, primero, de "e" con "c" - (e (c)) - y, a continuación, de "e" con "d" - (e (d))—, se sigue un efecto para nosotros crucial: el establecimiento 
de la relación (c (d)), tal cual se explicita en (27), un caso paralelo al comentado a propósito de (16):

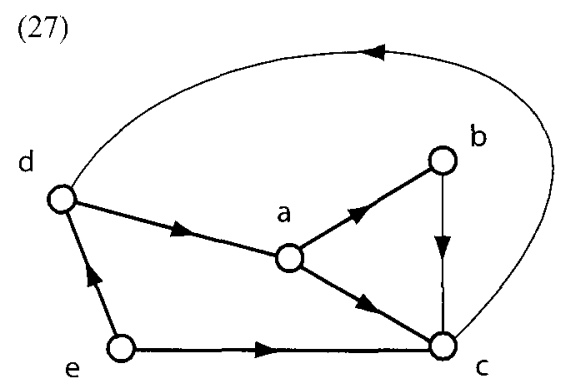

Esto significa que "c", que hasta ese momento no había contraído ninguna relación, pasa a relacionarse, no sólo con " $\mathrm{d}$ ", sino con todos los elementos con que éste a su vez se relaciona. Es decir, obtenemos las relaciones nuevas (c (d)), (c (a)) y (c (b)); también las relaciones (b (d)), en virtud del establecimiento previo de las relaciones (b (c)) y (c (d)), y (a (d)), en función del establecimiento de las relaciones (a (c)) y (c (d)).

Todo lo anterior tiene importantes implicaciones para nuestro modelo:

1. En primer lugar, el número de relaciones nuevas a que da lugar el establecimiento de la cadena (e (d)) no es el que resultaría esperable si la cadena (e (c)) no se hubiese creado previamente. En este último caso, el número de relaciones nuevas resultantes hubiese sido el de cuatro: (e (d)), (e (a)), (e (b)) y (e (c)). En cambio, al haberse establecido previamente (e (c)), lo que ocurre es que se generan automáticamente nueve nuevas relaciones: las ya mencionadas (e (d)), (e (a)) y (e (b)), más (c (d)), (c (a)), (c (b)), (b (d)), (b (a)) y (a (d)). Esto significa que el establecimiento de la cadena (e (c)) marca un punto de "inestabilidad" en el sistema capaz de provocar la aparición de un número significativamente mayor de relaciones del que sería esperable sin su establecimiento. En otras palabras, la cadena (e (c)) es responsable de una sobregeneración de relaciones por encima de lo previsible en la siguiente aplicación, por lo demás normal, de E.

2. En segundo lugar, una parte de estas relaciones resultan ser "imagen especular" de otras generadas anteriormente. Se refleja en el siguiente cuadro:

(28)

\begin{tabular}{|l|l|l|}
\hline relaciones nuevas: & $(\mathrm{c}(\mathrm{d}))(\mathrm{c}(\mathrm{a}))(\mathrm{c}(\mathrm{b}))(\mathrm{b}(\mathrm{d}))(\mathrm{b}(\mathrm{a}))(\mathrm{a}(\mathrm{d}))$ \\
\hline relaciones previamente existentes: $(\mathrm{d}(\mathrm{c}))(\mathrm{a}(\mathrm{c}))(\mathrm{b}(\mathrm{c}))(\mathrm{d}(\mathrm{b}))(\mathrm{a}(\mathrm{b}))(\mathrm{d}(\mathrm{a}))$ \\
\hline
\end{tabular}

Esto significa que el sistema pasa a proporcionar toda una serie de relaciones sintácticas aptas para el establecimiento de vínculos que impliquen la inversión de las relaciones de dominio establecidas con anterioridad, algo indudablemente provechoso a efectos gramaticales. Así, (c (d)) permite, por ejemplo, la inversión de la "superioridad" previamente existente del tema sobre el agente - de la que saca provecho una secuencia como (14)—. Esta inversión es la que permite que en secuencias como (29) el agente sirva como antecedente del elemento pronominal localizado en el tema: 


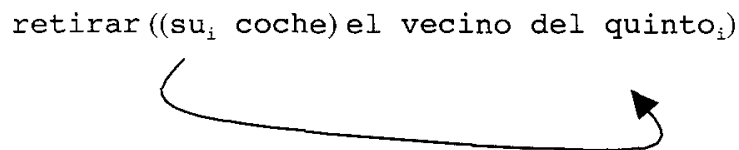

También puede servirnos para explicar ciertos efectos interpretativos relacionados con la "presuposición de existencia" asociada a las frases definidas en posición de sujeto. En Lorenzo (2000) se razona que, frente a la suposición más común, la idea de que las frases definidas en posición de sujeto "presuponen" la existencia de su referente no es del todo exacta. Resulta correcta en casos como (30a), oración formada a partir del predicado individual "ser un glotón", pero no en casos como (30b), formada a partir del predicado eventivo "presidir":

(30) a. El rey de Francia es un glotón.

b. El rey de Francia presidió la clausura de la última Eurocopa.

La diferencia fundamental entre uno y otro enunciado consiste en que mientras que del primero no es posible decir que se corresponda con una proposición ni verdadera ni falsa (dada la inexistencia del rey de Francia), de la segunda resulta perfectamente válido decir que corresponde con una proposición falsa (en la medida en que, de todos modos, el inexistente rey de Francia no estuvo presente en tal evento). De hecho, la versión negativa de esta última oración ("el rey de Francia no presidió la clausura de la última Eurocopa") nombra evidentemente una proposición verdadera, mientras que de la de la primera ("el rey de Francia no es un glotón") cabe decir lo mismo que de su versión afirmativa: no se corresponde con una proposición verdadera ni con una falsa. Teniendo en cuenta todo lo anterior, parece que podemos concluir que la diferencia fundamental entre (30a) y (30b) radica en que en el segundo caso se afirma la existencia del sujeto (lo que determina la falsedad de la proposición, que lo representa como presente), mientras que en el segundo simplemente se presupone (lo que determina la "laguna veritativa" de la proposición). Podemos decir que (30b) es, por tanto, un enunciado "russelliano" (en el que se "afirma" la existencia del sujeto definido), mientras que el (30a) es un enunciado "strawsoniano" (en el que sólo se "presupone").

Creemos que la malla (27), junto con ciertas asunciones no problemáticas en el análisis de las oraciones en (30), puede servirnos para explicar este tipo de efectos. De acuerdo, entre otros, con Kratzer (1989) o Diesing (1992), los sintagmas verbales están sujetos a una operación de "cierre existencial" que, desde nuestro punto de vista, se obtiene a través del encadenamiento de un operador existencial abstracto (" $\exists$ ") con el núcleo del predicado - en (27), por tanto, a través de $(d(a))$, es decir, $(\exists(V))$ - Esto nos da pie a considerar que la diferencia fundamental entre las interpretaciones de (30a) y de (30b) consiste en que en el primer caso el sujeto se interpreta a través del encadenamiento (c ( $(\mathrm{d}$ $(\mathrm{a}))))$ - es decir, (el rey de Francia $((\exists$ (ser un glotón $)))$ ) - , mientras que en el segundo se interpreta a través del encadenamiento $(\mathrm{d}((\mathrm{a}(\mathrm{c}))))$ - es decir, $(\exists((\mathrm{el}$ rey de Francia (presidir) $))$ )- - En este último caso sucede, por tanto, que el sujeto queda bajo el alcance del operador existencial, de modo que podemos entender que su existencia queda afirmada (y, por tanto, expuesta a refutación); en el primero, en cambio, el sujeto escapa al alcance 
del operador y su existencia queda únicamente presupuesta por familiaridad (en este caso, infundada) ${ }^{8}$.

3. Todo lo anterior nos encamina, además, hacia la conclusión más importante de toda esta sección. El modo como "e" se ha ensamblado (doblemente, en la medida en que está sujeto a un doble imperativo semántico) a la malla desarrollada hasta el momento de su inserción, sirve para dar cuenta de los efectos típicamente asociados a la operación transformacional del movimiento. No olvidemos que la justificación básica de la operación es la de permitir a un elemento (o a la propia estructura, según los modelos) la satisfacción de las diferentes condiciones a las que esté sometido, como, por ejemplo, recibir rol temático, de un lado, y entablar una relación de concordancia, de otro lado, en el caso de los argumentos externos 9 .

En la próxima sección tendremos ocasión de extendernos en algunos otros tipos de "movimiento" sintáctico y de comprobar que LMental no sólo los elimina, al derivar los efectos de la operación de movimiento de sus propiedades computacionales, sino que esta eliminación trae consigo otras consecuencias tan reseñables como la de poder renunciar a la distinción clásica entre operaciones de movimiento "explícito" (o "abierto") y de movimiento "implicito" (o "encubierto").

\section{Propiedades avanzadas de LMental: Derivando el "desplazamiento implícito" me- diante "ensamblaje"}

Consideremos las dos interpretaciones que es posible atribuir a una oración como (31):

(31) Alguien asistió a todas las clases.

(32) a. "una misma persona asistió a todas y cada una de las clases"

b. "al menos una persona (no necesariamente la misma) asistió a cada una de las clases"

La derivación de cada una de estas interpretaciones suele ser explicada como debida a una diferencia de alcance entre las frases cuantificadas (alguien y todas las clases) que aparecen en la oración: (32a) resulta de la atribución de alcance a la frase cuantificada existencialmente (alguien) sobre la cuantificada universalmente (todas las clases), mientras que (32b) resulta de la atribución de alcance contraria. Para una oración como (31) se observa que las dos atribuciones de alcance resultan posibles y que se escogen libremente, pero para otras oraciones lo que se aprecia es que tan sólo resulta posible una de ellas, en concreto, la que se corresponde con la disposición lineal de los cuantificadores en la oración (es decir,

8 Entendemos que la orientación de la interpretación hacia uno u otro de los encadenamientos es una propiedad léxica de los predicados respectivos. No olvidemos que LMental no hace más que proporcionar relaciones sintácticas aptas para su aprovechamiento semántico por parte de los elementos que participan en ellas.

9 De acuerdo con la "hipótesis del sujeto interno al SV" (véase, por ejemplo, Koopman y Sportiche 1991), plenamente incorporada al bagaje teórico del Programa Minimalista, el argumento externo obtiene su rol temático en [Spec, SV] y satisface su requisito de caso mediante el desplazamiento a [Spec, ST]. Asumiendo aquella misma hipótesis, Diesing (1992) maneja una ulterior operación de desplazamiento descendente en Forma Lógica para explicar efectos interpretativos que implican una relación de alcance del operador existencial sobre el argumento externo. 
alcance largo de la frase cuantificada existencialmente). Se puede observar en las oraciones (33) y (34):

(33) Alguien prometió a Juan asistir a todas las clases.

(34) Alguien expresó el deseo de asistir a todas las clases.

La estrategia de análisis tradicionalmente asumida por la gramática generativa para explicar que (31) pueda asociarse a una interpretación en que la relación de alcance contraviene la que inmediatamente se sigue de la disposición lineal de los cuantificadores (es decir, alcance largo de la frase cuantificada universalmente) consiste en la aplicación de una operación de movimiento "implícito" (es decir, en una fase de la derivación sin repercusión en el orden lineal establecido en la "fonologización") que desplaza el cuantificador universal a una posición desde la que manda-c y así pasa a ejercer alcance sobre el existencial. En esta línea de análisis, la imposibilidad de la interpretación con alcance largo del cuantificador universal en oraciones como (33) y (34) se explica como debida a las mismas razones que bloquean el movimiento explícito de operadores a partir de estructuras semejantes:

(35) *? ¿A qué clasesi prometió alguien a Juan asistir hi?

(36) *? ¿A qué clasesi expresó alguien el deseo de asistir $h_{i}$ ?

Esta tradición analítica ha conocido diferentes versiones a lo largo de las últimas décadas ${ }^{10}$, todas las cuales pueden considerarse, no obstante, como variantes sobre un mismo tema, cuya núcleo esencial se encuentra en la siguiente definición de "alcance" (véase May 1985: 5):

(37) El "alcance" de $\alpha$ es el conjunto de nodos que $\alpha$ manda-c en Forma Lógica,

donde "Forma Lógica" es el nivel de representación resultante de las aplicaciones de la operación de "muévase- $\alpha$ " sin repercusión fonológica y "mando-c" la relación que $\alpha$ mantiene con todos aquellos nodos estructurales a los que $\alpha$ no domina pero sí el primer nodo que domina a $\alpha$. Así, de acuerdo con el análisis May (1985), la adjunción ("implícita") de los cuantificadores al nodo oracional en una oración como (31), da lugar a una representación en Forma Lógica como la que se registra en (38):

(38) [o a todas las clases [o alguien $_{i}\left[o h_{i}\right.$ asistió $\left.\left.\left.h_{j}\right]\right]\right]$

En esta representación los dos cuantificadores se mandan-c mutuamente, en la medida en que el primer nodo que domina a cada uno de ellos (concretamente, $\mathrm{O}$ ) domina asimismo al otro ${ }^{11}$, de ahí que la oración (30) sea compatible con dos interpretaciones diferentes, en cada una de las cuales uno de los cuantificadores ejerce alcance sobre el otro.

Desde el punto de vista de este tipo de análisis, la adjunción implícita de los cuantificadores ("Elevación de Cuantificadores") es una operación que se efectúa de manera obligato-

10 Véase, por ejemplo, May (1977) y (1985) y Aoun y Li (1993), así como Huang (1995) y Hornstein (1995) para una visión de conjunto.

11 Resulta crucial para este tipo de análisis el supuesto de que en (37) los cuantificadores están dominados por diferentes segmentos de un mismo nodo oracional y no por diferentes nodos oracionales, de acuerdo con la distinción apuntada en Chomsky (1986: 7). 
ria y que se sujeta al mismo tipo de restricciones que otros tipos de movimiento explícito ${ }^{12}$. Así, lo que ocurre con (35) y con (36) es que la adjunción implícita al nodo oracional del cuantificador universal se ve bloqueada por la misma razón que el movimiento explícito del operador interrogativo en (35) y en (36), de modo que el cuantificador existencial ejerce alcance sobre aquel en Forma Lógica, pero no a la inversa.

Pasemos ahora a considerar qué tipo de análisis puede ofrecer LMental para las estructuras oracionales con cuantificación múltiple y si resulta capaz de explicar los contrastes interpretativos entre oraciones como (31), de un lado, y como (33) y (34) de otro lado. Lo primero que debemos notar es que la noción de "mando-c" no es un primitivo teórico de nuestro procedimiento, de manera que no podemos basar en ella la definición de "alcance". Si la sustituimos por la de "dominio estructural" (Dom), la definición resultante es la siguiente:

(39) El "alcance" de $\alpha$ es el conjunto de nodos a los que $\alpha$ domina estructuralmente.

Si elaboramos ahora el grafo correspondiente a la oración (30), lo que tenemos ocasión de observar es que las dos frases cuantificadas se dominan mutuamente, lo que explica la capacidad de cualquiera de ellas para ejercer alcance sobre la otra:

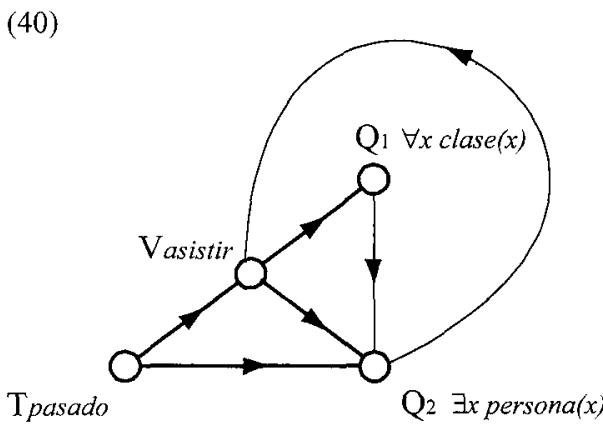

De un lado, la frase cuantificada universalmente $\left(\mathrm{Q}_{1}=\right.$ todas las clases $)$ domina a la frase cuantificada existencialmente $\left(\mathrm{Q}_{2}=\right.$ alguien $)$ como consecuencia de la relación que se establece automáticamente en función del encadenamiento previo de $\mathrm{V}$ (asistir) con $\mathrm{Q}_{1}$ y con

12 Hornstein (1995: Ch. 8) propone una variante del tipo de análisis que estamos considerando que ofrece la singularidad de eliminar la "Elevación de Cuantificadores", como un tipo de operación que se aplica especificamente sobre los cuantificadores, en favor del movimiento a las posiciones de especificador de la estructura funcional de la oración que, de acuerdo con el análisis de Chomsky (1993), se aplica sobre todos los argumentos. De acuerdo con Hornstein, la operación que tiene lugar en el nivel de Forma Lógica consiste, no en el movimiento de los argumentos cuantificados, sino en la elección ya sea de su posición de origen (en la estructura léxica de la oración) ya sea de su posición de destino (en la estructura funcional) como "locus" sobre el que se aplica su interpretación. De este modo, por ejemplo, la lectura (31b) se obtiene si el argumento cuantificado universalmente se interpreta en la estructura funcional y el cuantificado existencialmente en la léxica. El carácter unívoco de (32) y (33) se seguirá, según esta propuesta, del hecho de que en la estructura funcional de estas oraciones no existen posiciones en las que el constituyente cuantificado universalmente pueda encontrar acomodo. Puesto que la propuesta de Hornstein no evita la invocación de operaciones de movimiento para derivar las interpretaciones de estos tipos de oración, podemos considerarla como una variante más del tipo de análisis que nuestro trabajo se propone superar. 
$\mathrm{Q}_{2}$ de cara a la satisfacción de los imperativos semánticos del verbo relacionados con sus propiedades de selección argumental ${ }^{13}$. De otro lado, la frase cuantificada existencialmente pasa a dominar a la cuantificada universalmente una vez que se inserta $T$ (pasado) como introductor de la estructura básica de localización (BL) y se establecen las relaciones capaces de dar satisfacción a los imperativos semánticos a los que aquella está sujeta: en primer lugar, el encadenamiento con el argumento $Q_{2} y$, en segundo lugar, el encadenamiento con el predicado $V$, gracias al cual $Q_{2}$ entra en relación con y pasa a dominar estructuralmente a $Q_{1}$. En concreto, el encadenamiento de $T$ con $Q_{2}$ y con $V$ motiva que $Q_{2}$ se relacione automáticamente con $\mathrm{V}$, a su vez ya relacionado con $\mathrm{Q}_{1}$, de modo que $\mathrm{Q}_{2}$ pasa asimismo a relacionarse con $\mathrm{Q}_{1}{ }^{14}$. Obtenemos, por tanto, los efectos interpretativos propios de una oración con cuantificación múltiple sin la necesidad de apelar a ninguna operación de movimiento, sino como un efecto más de la aplicación normal del recurso computacional de LMental (esto es, Ensamblaje).

¿Qué es entonces lo que previene, si no es una restricción sobre el movimiento de constituyentes, que la frase cuantificada universalmente ejerza alcance sobre la cuantificada existencialmente en oraciones como (33) o (34)? Para entenderlo, conviene que observemos las propiedades formales del grafo correspondiente a una de estas oraciones. Sea, por ejemplo, la oración (33) y consideremos, en primer lugar, el grafo correspondiente a la cláusula de infinitivo (PRO asistir a todas las clases) subordinada al verbo principal (prometer), cuya estructura, reflejada en (41), no difiere de la (40):

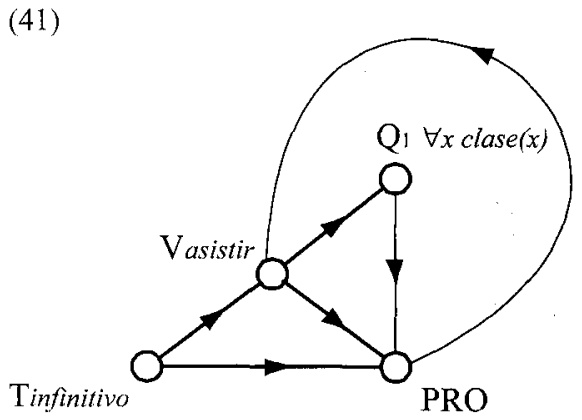

Como en el caso de (40), este grafo tiene como propiedad formal más relevante el que todos sus nodos, con la excepción del introductor de la BL T (infinitivo), se relacionan y dominan mutuamente. A la sección de un grafo cuyos nodos se domina mutuamente la denominaremos en adelante bucle. Un bucle es, por tanto, el efecto que se sigue de la incorporación al grafo de un introductor de BL y de la manera como debe satisfacer los imperativos semánticos a que se encuentra sujeto debido a la incidencia de los Principios $\Gamma$ y $\Sigma$. El bucle de (41), por tanto, incluye a todos los nodos del grafo salvo $T$ (infinitivo), tal cual se representa en (42): 


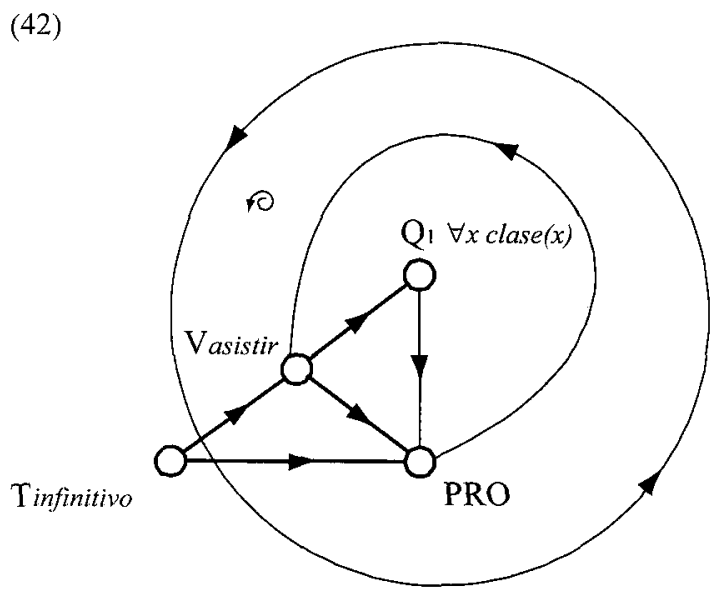

Pasemos ahora a considerar el resto de la configuración correspondiente al grafo de (33), reflejada en (43):

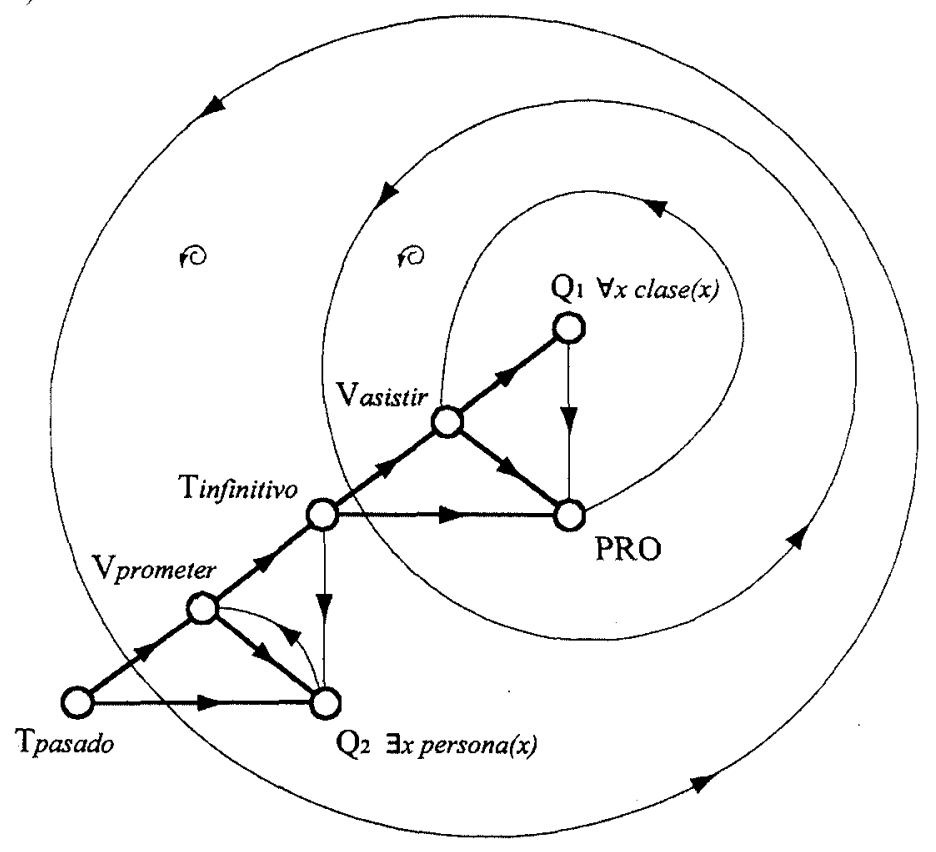

La propiedad formal más relevante para nosotros en este grafo se sigue de la condición de $\mathrm{T}$ (infinitivo) como punto de inserción para el establecimiento de la relación entre el verbo principal $\mathrm{V}$ (prometer) y el grafo (42), que es representación de uno de sus argumentos. $\mathrm{Al}$ ensamblar el introductor principal de BL T (pasado) y relacionarlo, primero, con el argumento $\mathrm{Q}_{2}$ (alguien) de $\mathrm{V}$ (prometer) y, después, con el propio predicado $\mathrm{V}$ (prometer) tiene 
automáticamente lugar la generación de un nuevo bucle: gracias a este segundo bucle, $\mathrm{Q}_{2}$ pasa a relacionarse con $\mathrm{V}$ (prometer) y, a través de esta relación, con $\mathrm{T}$ (infinitivo) y con la totalidad de los nodos con que éste se relaciona. Por tanto, $\mathrm{Q}_{2}$ (alguien) establece una relación y domina a $\mathrm{Q}_{1}$ (todas las clases), de modo que la frase cuantificada existencialmente puede ejercer alcance sobre la cuantificada universalmente. Sin embargo, la relación inversa no existe en el grafo (43). La explicación es que ninguno de los nodos de (42) se relaciona con $\mathrm{T}$ (infinitivo), debido precisamente a la exclusión de éste del bucle del que todos los demás forman parte $\left(\right.$ bucle $\left._{l}\right)$. Esto hace que T (infinitivo) actúe como un "tapón" que impide que ninguno de los nodos del bucle, pueda relacionarse con los nodos del bucle superior (bucle $e_{2}$ ). Así, la frase cuantificada universalmente (todas las clases), localizada en el bucle, no puede relacionarse con la cuantificada existencialmente (alguien), localizada en el bucle $y$, en consecuencia, no la domina ni puede ejercer alcance sobre ella.

\section{Consideraciones finales}

Concluiremos esta primera aproximación a LMental haciendo un repaso de sus principales virtudes como modelo del Sistema Computacional de la facultad humana del lenguaje bajo las premisas del minimalismo chomskyano. Las podemos agrupar en torno a tres de los temas o motivos fundamentales fijados en el programa de investigación minimalista:

1. Preferencia por soluciones altamente generales y mínimamente marcadas desde el punto de vista de su dedicación al cómputo de las expresiones verbales. En este sentido, LMental se atiene estrictamente al supuesto chomskyano (Chomsky 1995b) de que el carácter binario de las computaciones y el carácter asimétrico de las relaciones que se obtienen de ellas son reflejo de la naturaleza funcionalmente inespecífica o inmotivada del procedimiento, que opera con los principios más simples y generales concebibles.

2. Eliminación de "imperfecciones" gramaticales. La principal aportación de LMental a este respecto consiste en la eliminación de la operación de "desplazamiento" de constituyentes, precisamente señalada por Chomsky como una de las imperfecciones (esto es, desajustes con relación a las expectativas o necesidades de los sistemas de actuación) del Sistema Computacional de las lenguas naturales. Opinamos que, en lo tocante a este punto, LMental demuestra una superioridad teórica muy notable con relación a los posicionamientos de Chomsky al respecto, quien acaba por justificar y acomodar esta imperfección en el Sistema Computacional de las gramáticas (véase, por ejemplo, Chomsky 1998: 78-79 y 2000: 103).

3. Orientación derivacional de la idea de gramática. En este sentido, LMental consigue superar los "residuos" representacionales presentes incluso en las principales formulaciones y aplicaciones del Programa Minimalista, como el "etiquetado" de las proyecciones o la "persistencia" de las representaciones obtenidas en los sucesivos pasos de una derivación (véase Collins 2002). LMental permite trascender, además, la distinción entre operaciones "explícitas" e "implícitas", lo que juega a favor de la eliminación de los niveles de representación en que se consignan los resultados de unas y otras. La propia eliminación del desplazamiento, al evitar la necesidad de marcar posiciones como "origen" y "destino" de las operaciones, incide en la superación de cualquier residuo representacional en el modelo. 
Somos en todo caso conscientes de lo limitado de las aplicaciones que hemos podido llevar a cabo en esta primera presentación de LMental. Nuestra impresión general, a la vista de estas primeras aplicaciones, es que el procedimiento parece extremadamente adecuado para explicar los efectos tradicionalmente confiados a todo tipo de movimientos locales (Movimiento de SN, Elevación de Cuantificadores, etc.) y que tropezará con interesantes desafios para derivar las propiedades de las operaciones a larga distancia. Somos, en fin, plenamente conscientes de estar articulando un modelo del Sistema Computacional del lenguaje humano extremadamente poderoso y cuyas propiedades se nos escapan aún en buena medida. Explorarlas y fijarlas adecuadamente será el tema que nos ocupará en futuros trabajos.

\section{APÉNDICE.}

\section{Definición de los conceptos de "red", "malla" y "cadena".}

Red de relaciones de dominio estructural. Es la estructura relacional compleja que resulta de la iteración de $E$. A las relaciones que se dan entre los elementos de la red las denominamos "relaciones de dominio estructural" y permiten ver cómo están vinculados entre sí los elementos. Las relaciones de "dominio" no tienen sentido teórico (salvo desde un punto de vista estructural), ya que lo único relevante para el lenguaje es el contenido de las relaciones semánticas. Utilizamos la noción de "dominio" para explicar la habilitación de las relaciones semánticas.

Malla. La malla es una estructura isomórfica con una red, aunque considerada desde una perspectiva conceptual o semántica. Son sintácticas si las miramos desde el prisma de la red (un hecho relacional puro), pero semánticas si lo hacemos desde un punto de vista interno a la teoría. En nuestro caso, desde el punto de vista de la subordinación del sistema computacional a los dictados de los sistemas de pensamiento.

redes

A

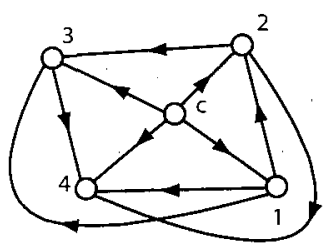

C

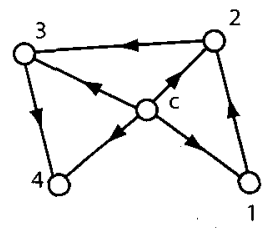

mallas

B

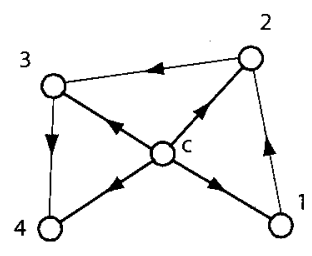

D

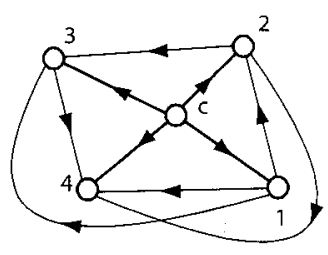


Tanto las redes como las mallas son isomorfas: tienen la misma estructura. Las redes pueden ser útiles para representar el número y dirección de las relaciones. Tanto (A) como (C) representan redes (de hecho, la misma red). No obstante, (C) es una representación más adecuada que (A), en la que parte de las relaciones especificadas son redundantes (por ejemplo, la relación (1(3)) está representada dos veces). Lo mismo pasa con las mallas (B) y (D). Las dos son correctas, pero la teóricamente válida es la malla (B). Las relaciones, al ser asimétricas, establecen una dirección o camino entre ellas (como se puede apreciar sin ninguna demostración, si aRc y $\mathrm{cRk} \Rightarrow \mathrm{aRk}$, es decir, que si "a" domina "c", y "c" domina " $k$ ", entonces "a" domina " $k$ "). Por tanto, la malla (D) representa redundantemente ciertas relaciones. Por otro lado, tanto una malla como (D) como una red como (A), dejan de representar el diferente carácter de las relaciones (locales o indirectas), relevante desde el punto de vista teórico asumido: por ejemplo, el que la relación (1 (3)) no sea una relación directa o local, sino que se establezca a través de la mediación de otra relación: (1 (2)) y $(2(3)) \Rightarrow(1(3))$.

Cadena. Es el conjunto de relaciones semánticas imperativas. Estas relaciones son relaciones locales y, por tanto, sólo se pueden generar mediante la aplicación de $E$. Una cadena es un subconjunto del conjunto de relaciones de una malla. Las cadenas sólo existen en las mallas (es decir, no existen antes de haber dado un sentido teórico a las redes).

\section{Referencias bibliográficas}

Aoun, J.; y A. Li, Y.-h. (1993): Syntax of Scope. Cambridge (MA), The MIT Press.

Chomsky, N. (1986): Barriers. Cambridge (MA), The MIT Press.

Chomsky, N. (1993): “A Minimalist Program for Linguistic Theory”. En Hale, K. y S.J. Keyser (eds.):

The View From Building 20. Essaysin Linguistics in Honor of Sylvain Bromberger. Cambridge (MA), The MIT Press, págs. 1-52 (incluido en Chomsky 1995a).

Chomsky, N. (1995a): The Minimalist Program. Cambridge (MA), The MIT Press.

Chomsky, N. (1995b): "Bare Phrase Structure". En Webelhuth, G. (ed.): Government and Binding Theory and the Minimalist Program. Oxford, Blackwell, págs. 383-439.

Chomsky, N. (1998): "Nuevos horizontes en el estudio del lenguaje". En Una aproximación naturalista a la mente y al lenguaje. Barcelona, Prensa Ibérica, págs. 67-85.

Chomsky, N. (2000): "Minimalist Inquiries: The Framework". En Martin, R., Michaels, D. y J. Uriagereka (eds.): Step by Step. Essays on Minimalist Syntax in Honor of Howard Lasnik. Cambridge (MA), The MIT Press, págs. 89-155.

Chomsky, N. (2005): “Three Factors in Language Design", Linguistic Inquiry, 36, págs. 1-22.

Collins, C. (2002): "Eliminating Labels". En Epstein, S. D. y T.D. Seely (eds.): Derivation and Representation in the Minimalist Program. Oxford, Blackwell, págs. 42-64.

Diesing, M. (1992): Indefinities. Cambridge(MA), The MIT Press.

Epstein, S. D., Groat, E., Kawashima, R. y H. Kitahara (1998): A Derivational Approach to Syntactic Relations. Oxford, Oxford University Press.

Hornstein, N. (1995): Logical Form. From GB to Minimalism. Oxford, Blackweil.

Huang, C.-T. J. (1995): "Logical Form". En Webelhuth, G. (ed.): Government and Binding Theory and the Minimalist Program: Principles and Parameters in Syntactic Theory. Oxford, Blackwell.

Koopman, H. y D. Sportiche (1991): "The Position of Subjects", Lingua, 85, págs. 211-258.

Kratzer, A. (1989): "Stage-Level and Individual-Level Predicates". En Bach, E., Kratzer, A. y B. Partee (eds.): Papers on Quantifications. Amherts, University of Massachusetts at Amherst.

Longa, V. M. y G. Lorenzo (2001): Derivación y representación. Su alternancia cíclica en la teoría de la gramática. Noia (A Coruña), Toxosoutos. 
Lorenzo, G. (2000): "Sujetos definidos, condición de existencia y tipos de predicados". Inédito (tema expuesto en los ejercicios de oposición al cuerpo de Profesores Titulares de Universidad). May, R. (1977): The Grammar of Quantification. MIT, Ph.D, dissertation. May, R. (1985): Logical Form. Its Structure and Derivation. Cambridge (MA), The MIT Press. 